\title{
Simple Adaptive Asymptotic Tracking Scheme for Parametric Strict-Feedback Nonlinear Systems with Additive Disturbance
}

\author{
Yanxia Wang, ${ }^{1}$ Zhengqiang Zhang, ${ }^{2} \mathrm{Lei} \mathrm{Li}^{2}$, and Junwei $\mathrm{Lu}^{3}$ \\ ${ }^{1}$ Qufu Normal University, Rizhao Campus, Rizhao 276826, China \\ ${ }^{2}$ School of Electrical Engineering and Automation, Qufu Normal University, Rizhao 276826, China \\ ${ }^{3}$ School of Electrical and Automation Engineering, Nanjing Normal University, 78 Bancang Street, Nanjing 210042, China \\ Correspondence should be addressed to Zhengqiang Zhang; qufuzzq@126.com
}

Received 19 October 2013; Accepted 9 December 2013

Academic Editor: Hao Shen

Copyright (C) 2013 Yanxia Wang et al. This is an open access article distributed under the Creative Commons Attribution License, which permits unrestricted use, distribution, and reproduction in any medium, provided the original work is properly cited.

\begin{abstract}
We consider the same class of systems in a previous paper, that is, a class of parametric strict-feedback nonlinear systems with additive disturbance. By using the backstepping technique, adaptive control algorithm is developed. Unlike the existing control schemes for systems with additive disturbance, no knowledge is assumed on two times continuous differentiability of the disturbance. Also, the developed backstepping controller does not require the uncertain parameters to be restricted within a known interval. Furthermore, overestimation of the system parameters is removed by employing the tuning functions method. It is shown that the proposed controller guarantees not only the stability in the sense of Lyapunov definition, but also the asymptotic tracking.
\end{abstract}

\section{Introduction}

Disturbance can be encountered in many physical systems. The control problem of such systems has received much attention in the past decades. For example, in [1], a class of singleinput-single-out linear systems with unmodelled dynamics and input and output disturbances were considered. A dynamic normalizing signal and a smooth switching- $\sigma$ function were introduced to design adaptive backstepping controller, and the mean-squared tracking error bound was established. In [2], adaptive control of the perturbed nonlinear output feedback systems was investigated. The Lyapunov functions with flat zone were employed, and the flatzone modifications were incorporated into the intermediate control functions. The output error was proved to converge to arbitrary small interval around zero. However, in most of control literature, asymptotic regulation or tracking could not be achieved, and the system stability was established only in the sense that all the closed-loop signals were bounded (see, e.g., [1-25]).

Recently, Cai et al. proposed a new class of adaptive controllers to deal with the control problem of nonlinear systems with disturbance [26-28]. Two special cases were firstly reported in the control conferences (see [26, 27]). In [26], the tracking problem for nonlinear parametric strict-feedback systems of relative degree two in the presence of matched additive disturbance were studied. In [27], the regulation problem for $n$ th-order nonlinear systems in the strictfeedback form was addressed. Then, in a more general case, the tracking control of strict-feedback $n$ th-order nonlinear systems with parametric uncertainties and additive disturbance was considered. The main contributions of these works were that continuous adaptive controllers were designed and asymptotic tracking performance was ensured. The basic control idea lied in the combination of robust control technique in [29] and sufficiently smooth projection operator [30]. While the mathematical manipulations in [26-28] are very elegant, the proposed control schemes are very complex. To be more important, in order to design the asymptotic tracking controller, several restrictive conditions are imposed to the controlled system, which will be stated as follows.

First, it is reasonable to require that the external disturbance is bounded. However, in [26-28], the disturbance was assumed to be two times continuous differentiable. Moreover, the first-order and second-order derivatives of 
the disturbance were required to be bounded by some known constants. These assumptions on the disturbance may be very limited in reality and in turn the practicality of the presented control schemes would be greatly reduced. Then, the system parameters must be constrained within some known interval. The purpose of making such assumption was to employ the smooth projection operator of [30]. On the one hand, the smooth projection algorithm can ensure the boundedness of the parameter estimates. On the other hand, the smoothness of such algorithm is critical for backstepping since the recursive design procedure requires multiple differentiations of the adaptive law. However, the lower and upper bounds of unknown system parameters may be not available to the designer in the control practice. Furthermore, in $[26,28]$, the control gain $\beta$ was selected to satisfy the following condition: $\beta>\|h(t)\|_{L_{\infty}}+\|\dot{d}(t)\|_{L_{\infty}}+1 / c_{n}\left(\|\dot{h}(t)\|_{L_{\infty}}+\|\ddot{d}(t)\|_{L_{\infty}}\right)$. It is easy to determine $\|\dot{d}(t)\|_{L_{\infty}}$ and $\|\ddot{d}(t)\|_{L_{\infty}}$, which is due to the system assumptions. However, the explicit expressions for $\|h(t)\|_{L_{\infty}}$ and $\|\dot{h}(t)\|_{L_{\infty}}$ were not provided in $[26,28]$. Actually, it is very difficult to specify the value of the design parameter $\beta$. As a result, the choice of $\beta$ in $[26,28]$ is only in the theoretical sense and it is not applicable to the practice. Finally, except for the last step of the recursive design, the unknown system parameters were repeatedly estimated in other steps of backstepping design. It can be computed that the total dynamic order of the parameter estimates is $(n-1) p$, where $n$ is the dimension of the controlled nonlinear system and $p$ is the dimension of the unknown system parameter. That is, the overestimation problem arose. It is well known that many parameter update laws will increase system complexity and implementation difficulty. So it is not desirable to design adaptive controller with too many parameter estimates in reality.

In this paper, we develop a simple adaptive backstepping control scheme for the same class of nonlinear systems as in [26-28], where the uncertain parameters and the matched external disturbance are considered simultaneously. The main contributions of the paper are as follows. (i) In our design, the external disturbance is only required to be bounded. Its upper bound is not necessary to be known and is estimated by designing adaptive law. (ii) The system parameters are not restricted to be within a known interval, and they are only assumed to be unknown constants. The projection algorithm or the smooth projection algorithm is not utilized in our design. Common adaptive laws without any modifications are constructed. (iii) The design parameters are not related to the external disturbance, the reference signal, the known system functions, and the initial conditions of the controlled system, and they can be freely chosen. Thus, the control performance of the closed-loop system can be greatly improved by choosing appropriate design constants. (iv) The tuning functions method [31] is employed to remove overestimation. Except for the estimate of the upper bound of the disturbance, the number of the parameter estimates is equal to that of the unknown system parameters. Besides showing the closed-loop stability in the Lyapunov sense, the tracking error is proven to converge to zero asymptotically.
This paper is organized as follows. After introducing the class of considered systems in Section 2, we present the main results of this paper in Section 3. In Section 4, an example with simulation to illustrate the effectiveness of the proposed design method is given. Finally, this paper is concluded by Section 5 .

\section{Problem Statement}

We consider the same class of SISO nonlinear systems as in [26-28], which are given as

$$
\begin{gathered}
\dot{x}_{i}=x_{i+1}+\varphi_{i}^{T}\left(x_{1}, \ldots, x_{i}\right) \theta, \quad i=1,2, \ldots, n-1, \\
\dot{x}_{n}=u+\varphi_{n}^{T}\left(x_{1}, x_{2}, \ldots, x_{n}\right) \theta+d(t),
\end{gathered}
$$

where $x_{1}, x_{2}, \ldots, x_{n}$, are system states, $u$ is control input, $\varphi_{i} \in R^{p}, i=1,2, \ldots, n$, are known and smooth nonlinear functions, $\theta \in R^{p}$ is unknown constant vector, and $d(t)$ is bounded additive disturbance. The objective is to enable the output $y=x_{1}$ to follow a desired trajectory $y_{r}$, while all the closed-loop signals remain bounded.

Assumption 1. The reference signal $y_{r}$ and its first $n$ derivatives are known and bounded.

To prove the asymptotic convergence of the tracking error, the following lemma is used.

Lemma 2 (Barbalat's lemma [32]). Let $f:[0, \infty) \mapsto R$. If $f, \dot{f} \in L_{\infty}$ and $f \in L_{2}$, then $f(t) \rightarrow 0$ as $t \rightarrow \infty$.

Remark 3. When $d(t)=0$, the system (1) is the standard parametric strict-feedback nonlinear system. The disturbance $d(t)$ appears in the same equation as the control input $u$. That is, the matched disturbance is considered. Assumptions (A2) and (A3) in [26-28] are removed in this paper. We will design the asymptotic adaptive controller under the relaxed conditions.

\section{Main Results}

In our design, the first $(n-1)$ steps are the same as those in common adaptive backstepping control with tuning functions. To avoid repetition, the design steps are summarized as follows (see [31, Page 158, Table 4.1, (4.189)-(4.192)]).

$$
\begin{aligned}
& \text { Step i. } i=1,2, \ldots, n-1, \\
& \begin{aligned}
z_{i} & =x_{i}-y_{r}^{(i-1)}-\alpha_{i-1}, \\
\alpha_{i}\left(\bar{x}_{i}, \bar{y}_{r}^{(i-1)}, \widehat{\theta}\right)= & -z_{i-1}-c_{i} z_{i}-\omega_{i}^{T} \widehat{\theta} \\
& +\sum_{j=1}^{i-1}\left(\frac{\partial \alpha_{i-1}}{\partial x_{j}} \cdot x_{j+1}+\frac{\partial \alpha_{i-1}}{\partial y_{r}^{(j-1)}} y_{r}^{(j)}\right) \\
& +\frac{\partial \alpha_{i-1}}{\partial \widehat{\theta}} \Gamma \tau_{i}+\sum_{j=2}^{i-1} z_{j} \frac{\partial \alpha_{j-1}}{\partial \widehat{\theta}} \Gamma \omega_{i},
\end{aligned}
\end{aligned}
$$




$$
\begin{gathered}
\tau_{i}\left(\bar{x}_{i}, \bar{y}_{r}^{(i-1)}, \widehat{\theta}\right)=\tau_{i-1}+\omega_{i} z_{i}, \\
\omega_{i}\left(\bar{x}_{i}, \bar{y}_{r}^{(i-2)}, \widehat{\theta}\right)=\varphi_{i}-\sum_{j=1}^{i-1} \frac{\partial \alpha_{i-1}}{\partial x_{j}} \varphi_{j}, \\
\bar{x}_{i}=\left[x_{1}, x_{2}, \ldots, x_{i}\right]^{T}, \\
\bar{y}_{r}^{(i)}=\left[y_{r}, \dot{y}_{r}, \ldots, y_{r}^{(i)}\right]^{T},
\end{gathered}
$$

where $y_{r}^{(0)}=y_{r}, z_{i}$ is error variable, $z_{0}=0, \alpha_{i}$ is virtual control law, $\alpha_{0}=0, \tau_{i}$ is tuning function, $\tau_{0}=0, \widehat{\theta}$ is the estimate of $\theta$ with the estimation error $\widetilde{\theta}=\theta-\widehat{\theta}, c_{i}>0$ is design constant, and $\Gamma$ is adaptive gain. In the above steps, the Lyapunov functions are, respectively, defined as

$$
\begin{gathered}
V_{1}=\frac{1}{2} z_{1}^{2}+\frac{1}{2} \widetilde{\theta}^{T} \Gamma^{-1} \widetilde{\theta}, \\
V_{i}=V_{i-1}+\frac{1}{2} z_{i}^{2}, \quad i=2, \ldots, n-1,
\end{gathered}
$$

and the derivative of $V_{n-1}$ is governed by

$$
\begin{aligned}
\dot{V}_{n-1}= & -\sum_{j=1}^{n-1} c_{j} z_{j}^{2}+z_{n-1} z_{n}-\widetilde{\theta}^{T}\left(\Gamma^{-1} \dot{\hat{\theta}}-\tau_{n-1}\right) \\
& +\sum_{j=2}^{n-1} z_{j} \frac{\partial \alpha_{j-1}}{\partial \widehat{\theta}}\left(\Gamma \tau_{n-1}-\dot{\hat{\theta}}\right) .
\end{aligned}
$$

The detailed deductions are similar to the corresponding parts in Section 4.2 of [31] (see [31, Page 140-146, (4.87)(4.131)]). In this section, only the last step of the design, that is, Step n, is elaborated in detail.

Step $n$. Define

$$
z_{n}=x_{n}-y_{r}^{(n-1)}-\alpha_{n-1} .
$$

From (1) and (10), we obtain

$$
\begin{aligned}
\dot{z}_{n}= & u+\varphi_{n}^{T} \theta+d(t)-y_{r}^{(n)}-\sum_{j=1}^{n-1} \frac{\partial \alpha_{n-1}}{\partial x_{j}} \\
& \cdot\left(\varphi_{j}^{T} \theta+x_{j+1}\right)-\sum_{j=1}^{n-1} \frac{\partial \alpha_{n-1}}{\partial y_{r}^{(j-1)}} y_{r}^{(j)}-\frac{\partial \alpha_{n-1}}{\partial \hat{\theta}} \dot{\hat{\theta}} \\
= & u+\omega_{n}^{T} \theta-y_{r}^{(n)}-\sum_{j=1}^{n-1} \frac{\partial \alpha_{n-1}}{\partial x_{j}} x_{j+1} \\
& -\sum_{j=1}^{n-1} \frac{\partial \alpha_{n-1}}{\partial y_{r}^{(j-1)}} y_{r}^{(j)}-\frac{\partial \alpha_{n-1}}{\partial \widehat{\theta}}+d(t),
\end{aligned}
$$

where

$$
\omega_{n}=\varphi_{n}-\sum_{j=1}^{n-1} \frac{\partial \alpha_{n-1}}{\partial x_{j}} \varphi_{j}
$$

We define the Lyapunov function as

$$
\begin{aligned}
V_{n} & =V_{n-1}+\frac{1}{2} z_{n}^{2}+\frac{1}{2} \gamma^{-1}{\widetilde{\bar{d}}^{2}}^{n} \\
& =\frac{1}{2} \sum_{j=1}^{n} z_{j}^{2}+\frac{1}{2} \widetilde{\theta}^{T} \Gamma^{-1} \tilde{\theta}+\frac{1}{2} \gamma^{-1} \widetilde{\bar{d}}^{2},
\end{aligned}
$$

where

$$
\begin{gathered}
\widetilde{\bar{d}}=\bar{d}-\widehat{\bar{d}}, \\
\bar{d}=\sup _{t \geq 0}|d(t)|,
\end{gathered}
$$

$\bar{d}$ denotes the upper bound of $d(t), \widehat{\bar{d}}$ is the estimate of $\bar{d}, \widetilde{\bar{d}}$ is parameter estimation error, and $\gamma$ is adaptive gain. With (9) and (11), the derivative of $V_{n}$ is given by

$$
\begin{aligned}
\dot{V}_{n}= & -\sum_{j=1}^{n-1} c_{j} z_{j}^{2}-\widetilde{\theta}^{T}\left(\Gamma^{-1} \dot{\hat{\theta}}-\tau_{n-1}\right)+\sum_{j=2}^{n-1} z_{j} \frac{\partial \alpha_{j-1}}{\partial \widehat{\theta}} \\
\cdot\left(\Gamma \tau_{n-1}-\dot{\hat{\theta}}\right)+z_{n}\left(z_{n-1}+u+\omega_{n}^{T} \theta-y_{r}^{(n)}\right. & \\
& -\sum_{j=1}^{n-1} \frac{\partial \alpha_{n-1}}{\partial x_{j}} x_{j+1}-\sum_{j=1}^{n-1} \frac{\partial \alpha_{n-1}}{\partial y_{r}^{(j-1)}} y_{r}^{(j)} \\
& \left.-\frac{\partial \alpha_{n-1} \dot{\hat{\theta}}}{\partial \hat{\theta}}\right)+z_{n} d(t)-\gamma^{-1} \simeq \dot{\bar{d}}
\end{aligned}
$$

By introducing

$$
\tau_{n}=\tau_{n-1}+\omega_{n} z_{n}
$$

and noting

$$
\theta=\widehat{\theta}+\tilde{\theta}
$$

(15) can be rewritten as

$$
\begin{aligned}
\dot{V}_{n}= & -\sum_{j=1}^{n-1} c_{j} z_{j}^{2}-\widetilde{\theta}^{T}\left(\Gamma^{-1} \dot{\hat{\theta}}-\tau_{n}\right)+\sum_{j=2}^{n-1} z_{j} \frac{\partial \alpha_{j-1}}{\partial \hat{\theta}} \\
\cdot\left(\Gamma \tau_{n-1}-\dot{\hat{\theta}}\right)+z_{n}\left(z_{n-1}+u+\omega_{n}^{T} \hat{\theta}-y_{r}^{(n)}\right. & -\sum_{j=1}^{n-1} \frac{\partial \alpha_{n-1}}{\partial x_{j}} x_{j+1}-\sum_{j=1}^{n-1} \frac{\partial \alpha_{n-1}}{\partial y_{r}^{(j-1)}} y_{r}^{(j)} \\
& \left.-\frac{\partial \alpha_{n-1} \dot{\hat{\theta}}}{\partial \widehat{\theta}}\right)+z_{n} d(t)-\gamma^{-1} \widetilde{\bar{d}} \dot{\bar{d}}
\end{aligned}
$$


Due to

$$
\begin{gathered}
\Gamma \tau_{n-1}-\dot{\hat{\theta}}=\Gamma \tau_{n-1}-\Gamma \tau_{n}+\Gamma \tau_{n}-\dot{\hat{\theta}} \\
=-\Gamma \omega_{n} z_{n}+\Gamma \tau_{n}-\dot{\hat{\theta}}, \\
z_{n} d(t) \leq\left|z_{n}\right| \bar{d},
\end{gathered}
$$

we have

$$
\begin{gathered}
\dot{V}_{n} \leq-\sum_{j=1}^{n-1} c_{j} z_{j}^{2}-\widetilde{\theta}^{T}\left(\Gamma^{-1} \dot{\hat{\theta}}-\tau_{n}\right)+\sum_{j=2}^{n-1} z_{j} \frac{\partial \alpha_{j-1}}{\partial \widehat{\theta}} \\
\cdot\left(\Gamma \tau_{n}-\dot{\hat{\theta}}\right)+z_{n}\left(z_{n-1}+u+\omega_{n}^{T} \widehat{\theta}-y_{r}^{(n)}\right. \\
\quad-\sum_{j=1}^{n-1} \frac{\partial \alpha_{n-1}}{\partial x_{j}} x_{j+1}-\sum_{j=1}^{n-1} \frac{\partial \alpha_{n-1}}{\partial y_{r}^{(j-1)}} y_{r}^{(j)} \\
+\left|z_{n}\right| \bar{d}-\gamma^{-1} \simeq \dot{\bar{d}} .
\end{gathered}
$$

Then, adaptive control law is designed as

$$
\begin{gathered}
u=-z_{n-1}-c_{n} z_{n}-\omega_{n}^{T} \widehat{\theta}+y_{r}^{(n)}+\sum_{j=1}^{n-1} \frac{\partial \alpha_{n-1}}{\partial x_{j}} x_{j+1} \\
+\sum_{j=1}^{n-1} \frac{\partial \alpha_{n-1}}{\partial y_{r}^{(j-1)}} y_{r}^{(j)}+\sum_{j=2}^{n-1} z_{j} \frac{\partial \alpha_{j-1}}{\partial \widehat{\theta}} \Gamma \omega_{n} \\
+\frac{\partial \alpha_{n-1}}{\partial \widehat{\theta}} \Gamma \tau_{n}-\frac{z_{n} \widehat{\bar{d}}^{2}}{\left|z_{n}\right| \widehat{\bar{d}}+\delta(t)} \\
\dot{\hat{\theta}}=\Gamma \tau_{n}, \\
\dot{\bar{d}}=\gamma\left|z_{n}\right|
\end{gathered}
$$

where $c_{n}$ is positive design constant, $\widehat{\bar{d}}(0) \geq 0$, and $\delta(t)$ is a time-varying function and satisfies

$$
\delta(t)>0, \quad \int_{0}^{t} \delta(\tau) d \tau \leq \bar{\delta}
$$

with positive constant $\bar{\delta}$.

Remark 4. It is noted that the first $(n-1)$ steps of the control design are the same as those in the standard backstepping procedure [31]. The difference between the tuning functions design of [31] and our scheme lies in the final step of the recursive design. In (21), the additional term $-z_{n} \widehat{\bar{d}}^{2} /\left(\left|z_{n}\right| \widehat{\bar{d}}+\delta(t)\right)$ is incorporated into the actual control law in order to suppress the influence of the disturbance on the closed-loop stability. Condition (24) implies that $\delta(t)$ is a positive time-varying integral function, which will play an important role in the subsequent stability analysis.

Substituting (21)-(23) into (20) yields

$$
\begin{aligned}
\dot{V}_{n} & \leq-\sum_{j=1}^{n} c_{j} z_{j}^{2}-\frac{z_{n}^{2 \hat{\bar{d}}^{2}}}{\left|z_{n}\right| \hat{\bar{d}}+\delta(t)}+\left|z_{n}\right| \widehat{\bar{d}} \\
& =-\sum_{j=1}^{n} c_{j} z_{j}^{2}+\frac{\left|z_{n}\right| \widehat{\bar{d}} \delta(t)}{\left|z_{n}\right| \widehat{\bar{d}}+\delta(t)} .
\end{aligned}
$$

In view of positive initial condition in (23) (see below (23)), we have $\widehat{\bar{d}}(t) \geq 0, t \geq 0$. By using the inequality $a b /(a+b) \leq b$, for all $a \geq 0, b>0$, (25) becomes

$$
\dot{V}_{n} \leq-\sum_{j=1}^{n} c_{j} z_{j}^{2}+\delta(t) .
$$

Integrating (26) on $[0, t]$ and using (24), we get

$$
\begin{aligned}
V_{n}(t) & +\sum_{j=1}^{n} \int_{0}^{t} c_{j} z_{j}^{2}(\tau) d \tau \\
& \leq V_{n}(0)+\int_{0}^{t} \delta(\tau) d \tau \leq V_{n}(0)+\bar{\delta}
\end{aligned}
$$

From (13) and (27), it follows that $V_{n}$ and thus $z_{j}, j=1$, $\ldots, n, \widehat{\theta}, \widehat{\bar{d}}$ are bounded. From (2) and (10), we have

$$
x_{i}=z_{i}+y_{r}^{(i-1)}+\alpha_{i-1}, \quad i=1, \ldots, n .
$$

According to Assumption 1 and the boundedness of $z_{1}, x_{1}$ is bounded. Since $\alpha_{1}$ is a smooth function of $x_{1}, y_{r}$, and $\hat{\theta}$, we conclude that $\alpha_{1}$ is bounded. Let $i=2$ in (28). We obtain the boundedness of $x_{2}$. By expecting the expressions in (3) and (4) with $i=2$, it is shown that $\alpha_{2}$ is bounded. Let $i=3$ in (28). Then, we have the boundedness of $x_{3}$. Continuing in this way, we can prove that $\alpha_{3}, x_{4}, \ldots, \alpha_{n-1}, x_{n}$, are bounded. Noting

$$
-\frac{z_{n} \hat{\bar{d}}^{2}}{\left|z_{n}\right| \widehat{\bar{d}}+\delta(t)} \leq \frac{\left|z_{n} \hat{\bar{d}}\right|}{\left|z_{n}\right| \widehat{\bar{d}}+\delta(t)}|\widehat{\bar{d}}| \leq|\widehat{\bar{d}}|
$$

and using the boundedness of $x_{n}$, we prove that $u$ in (21) is bounded.

Furthermore, differentiating (2) with $i=1$ gives

$$
\dot{z}_{1}=\dot{x}_{1}-\dot{y}_{r}=x_{2}+\varphi_{1}^{T} \theta-\dot{y}_{r} .
$$

Using the boundedness of all the closed-loop signals and Assumption 1, we know that $\dot{z}_{1} \in L_{\infty}$. Noting (27), we get

$$
\sum_{j=1}^{n} \int_{0}^{t} c_{j} z_{j}^{2}(\tau) d \tau \leq V_{n}(0)+\bar{\delta}
$$


which particularly implies that

$$
\int_{0}^{t} z_{1}^{2}(\tau) d \tau \leq \frac{1}{c_{1}}\left(V_{n}(0)+\bar{\delta}\right) .
$$

That is, $z_{1} \in L_{2}$. Thus, by Lemma 2, we obtain that $\lim _{t \rightarrow \infty} z_{1}(t)=0$.

The above facts prove the following results.

Theorem 5. Suppose that the proposed design procedure is applied to system (1). Then the boundedness of all signals in the resulting closed-loop system is guaranteed and asymptotic tracking is achieved.

\section{Simulation}

In this section, we illustrate the aforementioned methodologies to the same example system as that in [26], which is described as

$$
\begin{gathered}
\dot{x}_{1}=x_{2}+\varphi_{1}^{T}\left(x_{1}\right) \theta \\
\dot{x}_{2}=u+\varphi_{2}^{T}(x) \theta+d(t),
\end{gathered}
$$

where $\theta=[2,1]^{T}$ is assumed to be unknown constant, $\varphi_{1}, \varphi_{2}$, and $d(t)$ are, respectively, set to be

$$
\varphi_{1}=\left[\begin{array}{c}
-x_{1}^{2} \\
\frac{1}{4} x_{1}^{3}
\end{array}\right], \quad \varphi_{2}=\left[\begin{array}{c}
-x_{1}^{2} \\
\frac{1}{4} x_{2}^{3}
\end{array}\right], \quad d(t)=0.1 \sin (t) .
$$

The desired trajectory is $y_{r}=0.1 \sin (t)\left(1-e^{-0.5 t^{2}}\right)$. The design procedure in Section 3 is as follows.

Step 1. Consider

$$
\begin{gathered}
z_{1}=x_{1}-y_{r}, \quad \omega_{1}=\varphi_{1}, \\
\alpha_{1}=-c_{1} z_{1}-\omega_{1}^{T} \hat{\theta}, \quad \tau_{1}=\omega_{1} z_{1} .
\end{gathered}
$$

Step 2. Then

$$
\begin{gathered}
z_{2}=x_{2}-\dot{y}_{r}-\alpha_{1}, \\
\omega_{2}=\varphi_{2}-\frac{\partial \alpha_{1}}{\partial x_{1}} \varphi_{1}, \quad \tau_{2}=\tau_{1}+z_{2} \omega_{2} \\
u=-z_{1}-c_{2} z_{2}-\omega_{2}^{T} \hat{\theta}+y_{r}^{(2)}+\frac{\partial \alpha_{1}}{\partial x_{1}} x_{2} \\
+\frac{\partial \alpha_{1}}{\partial y_{r}} \dot{y}_{r}+\frac{\partial \alpha}{\partial \widehat{\theta}} \Gamma \tau_{2}-\frac{z_{2} \widehat{\bar{d}}^{2}}{\left|z_{2}\right| \widehat{\bar{d}}+\delta(t)} \\
\dot{\hat{\theta}}=\Gamma \tau_{2}, \quad \dot{\bar{d}}=\gamma\left|z_{2}\right| .
\end{gathered}
$$

The simulation is carried out with the following choices:

$$
\begin{gathered}
c_{1}=8, \quad c_{2}=1, \quad \Gamma=\operatorname{diag}\{1,1\}, \\
\gamma=0.5, \quad \delta(t)=0.01 e^{-0.01 t} .
\end{gathered}
$$

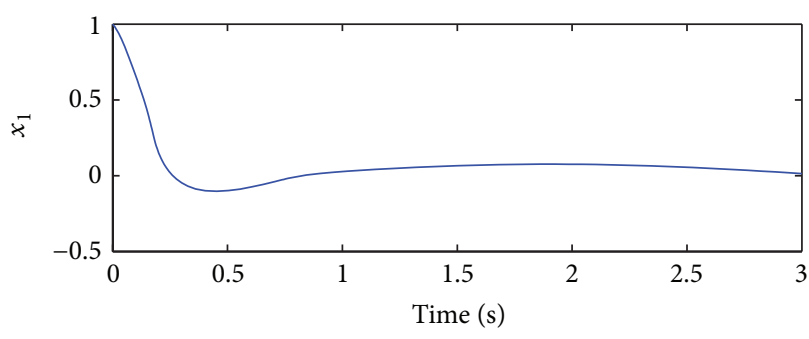

(a)

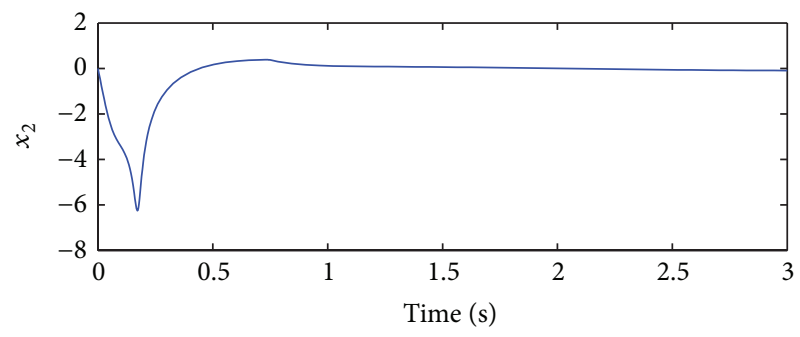

(b)

FIGURE 1: Plant states $x_{1}, x_{2}$.

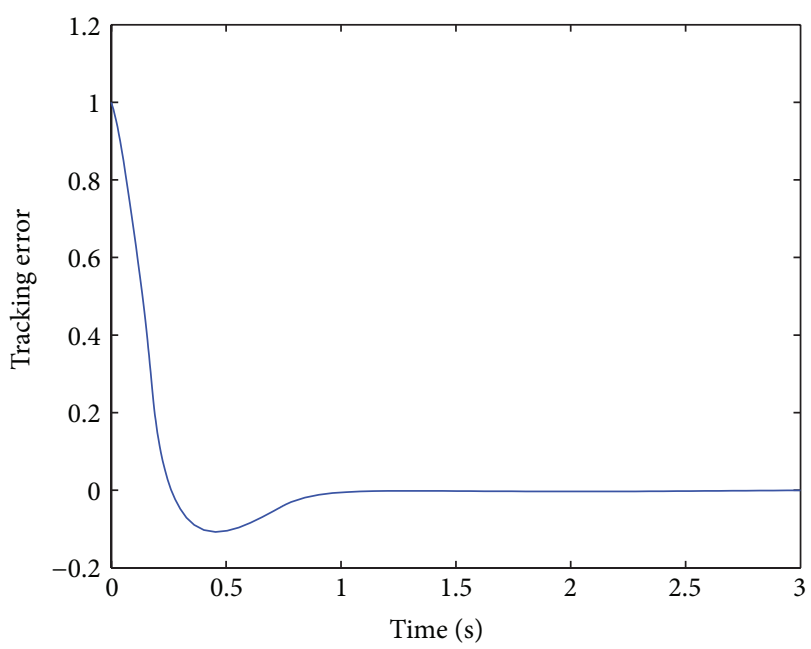

FIGURE 2: Tracking error $z_{1}$.

The initial conditions are set to be

$$
\begin{gathered}
x_{1}(0)=1, \quad x_{2}(0)=0, \\
\widehat{\theta}(0)=[1,-1]^{T}, \quad \widehat{\bar{d}}(0)=2 .
\end{gathered}
$$

The system responses are shown in Figures 1, 2, 3, and 4. From the simulation results, we observe that asymptotic tracking is achieved in the presence of external disturbance, and plant states, parameter estimate, and control effort are all bounded.

\section{Conclusions}

In this paper, we present an adaptive backstepping control design for a class of nonlinear systems with additive disturbance. Unlike some existing control schemes, the proposed 


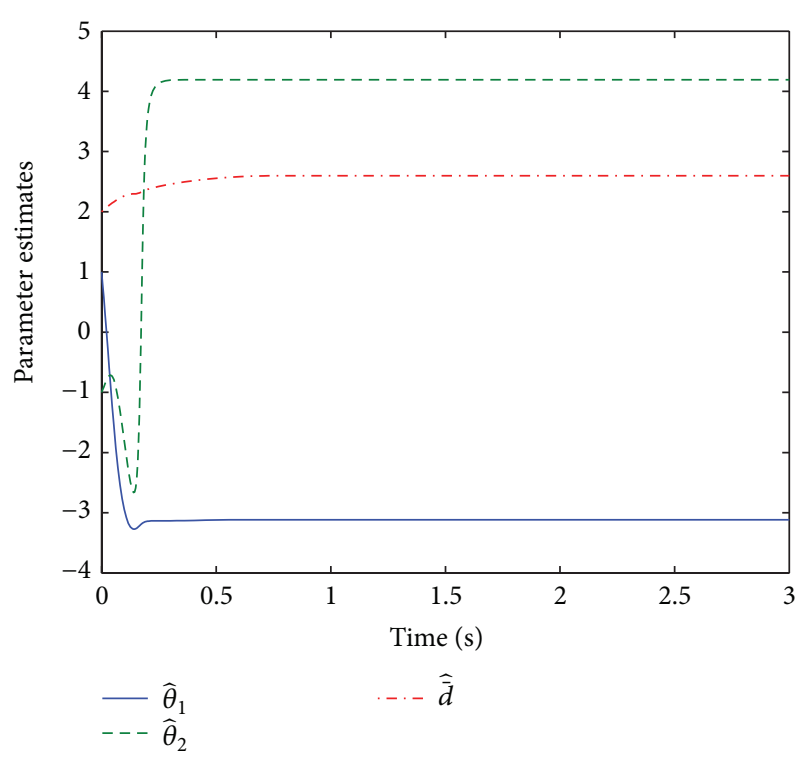

Figure 3: Parameter estimates $\widehat{\theta}=\left[\widehat{\theta}_{1}, \widehat{\theta}_{2}\right]^{T}, \widehat{\bar{d}}$.

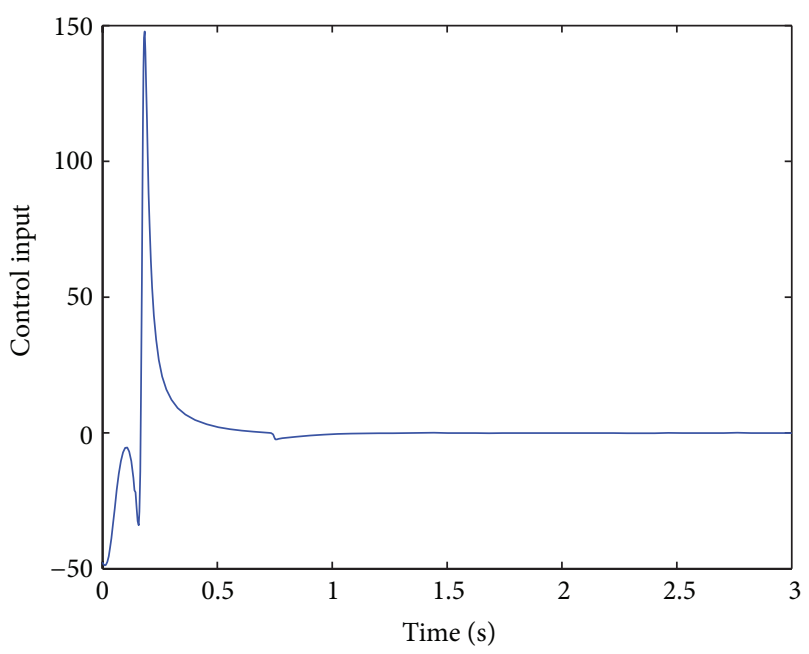

FIGURE 4: Control input $u$.

backstepping control does not require the knowledge on the differentiability of the disturbance and the known interval of the model parameters. In our design, a positive time-varying integral function is involved and can ensure perfect tracking. The overestimation problem is solved by introducing the tuning functions. Simulation results are provided to illustrate the effectiveness of our scheme.

\section{Acknowledgments}

This work was supported by the National Natural Science Foundation of China under Grants 61104007, 61203320, 61273123, and 61374090, China Postdoctoral Science Foundation funded project under Grant 2012M511465, Shandong Postdoctoral Science Foundation funded project under Grant 201203031, the project supported by the Young Core
Instructor, and Domestic Visitor Foundation from the Education Department of Shandong Province, China.

\section{References}

[1] Y. Zhang and P. A. Ioannou, "Robustness and performance of a modified adaptive backstepping controller," International Journal of Adaptive Control and Signal Processing, vol. 12, no. 3, pp. 247-265, 1998.

[2] Z. Ding and X. Ye, "A flat-zone modification for robust adaptive control of nonlinear output feedback systems with unknown high-frequency gains," IEEE Transactions on Automatic Control, vol. 47, no. 2, pp. 358-363, 2002.

[3] M. M. Polycarpou and P. A. Ioannou, "A robust adaptive nonlinear control design," Automatica, vol. 32, no. 3, pp. 423-427, 1996.

[4] R. A. Freeman, M. Krstić, and P. V. Kokotović, "Robustness of adaptive nonlinear control to bounded uncertainties," Automatica, vol. 34, no. 10, pp. 1227-1230, 1998.

[5] Z. Ding, "Almost disturbance decoupling of uncertain nonlinear output feedback systems," IEE Proceedings of Control Theory and Applications, vol. 146, no. 2, pp. 220-226, 1999.

[6] Z. Ding, "Analysis and design of robust adaptive control for nonlinear output feedback systems under disturbances with unknown bounds," IEE Proceedings, vol. 147, no. 6, pp. 655-663, 2000.

[7] B. Yao and M. Tomizuka, "Adaptive robust control of SISO nonlinear systems in a semi-strict feedback form," Automatica, vol. 33, no. 5, pp. 893-900, 1997.

[8] B. Yao and A. Palmer, "Indirect adaptive robust control of SISO nonlienr systems in semi-strict feedback forms," in Proceedings of the 15th IFAC World Congress, pp. 1-6, 2002.

[9] F. Hong, S. S. Ge, B. Ren, and T. H. Lee, "Robust adaptive control for a class of uncertain strict-feedback nonlinear systems," International Journal of Robust and Nonlinear Control, vol. 19, no. 7, pp. 746-767, 2009.

[10] R. Marino and P. Tomei, "Robust adaptive state-feedback tracking for nonlinear systems," IEEE Transactions on Automatic Control, vol. 43, no. 1, pp. 84-89, 1998.

[11] R. Marino and P. Tomei, "Adaptive tracking and disturbance rejection for uncertain nonlinear systems," IEEE Transactions on Automatic Control, vol. 50, no. 1, pp. 90-95, 2005.

[12] V. O. Nikiforov, "Adaptive non-linear tracking with complete compensation of unknown disturbances," European Journal of Control, vol. 4, no. 2, pp. 132-139, 1998.

[13] H. E. Psillakis and A. T. Alexandridis, "Adaptive neural tracking for a class of SISO uncertain and stochastic nonlinear systems," in Proceedings of the 44th IEEE Conference on Decision and Control, and the European Control Conference (CDC-ECC '05), pp. 7822-7827, December 2005.

[14] H. Wang, B. Chen, X. Liu, K. Liu, and C. Lin, "Robust adaptive fuzzy tracking control for pure-feedback stochastic nonlinear systems with input constraints," IEEE Transactions on Cybernetics, vol. 43, no. 6, pp. 2093-2104, 2013.

[15] Z. Zhang, S. Xu, Y. Guo, and Y. Chu, "Robust adaptive outputfeedback control for a class of nonlinear systems with timevarying actuator faults," International Journal of Adaptive Control and Signal Processing, vol. 24, no. 9, pp. 743-759, 2010.

[16] Z. Zhang, S. Xu, and H. Shen, "Reduced-order observer-based output-feedback tracking control of nonlinear systems with 
state delay and disturbance," International Journal of Robust and Nonlinear Control, vol. 20, no. 15, pp. 1723-1738, 2010.

[17] Z. Zhang, J. Lu, and S. Xu, "Tuning functions-based robust adaptive tracking control of a class of nonlinear systems with time delays," International Journal of Robust and Nonlinear Control, vol. 22, no. 14, pp. 1631-1646, 2012.

[18] S. He, Z. Ding, and F. Liu, "Output regulation of a class of continuous-time Markovian jumping systems," Signal Processing, vol. 93, no. 2, pp. 411-419, 2013.

[19] S. He and F. Liu, "Finite-time $\mathrm{H}$ control of nonlinear jump systems with time-delays via dynamic observer-based state feedback," IEEE Transactions on Fuzzy Systems, vol. 20, no. 4, pp. 605-614, 2012.

[20] S. He and F. Liu, "Filtering-based robust fault detection of fuzzy jump systems," Fuzzy Sets and Systems, vol. 185, no. 1, pp. 95-110, 2011.

[21] H. Shen, X. Huang, and Z. Wang, "Fuzzy dissipative control for nonlinear Markovian jump systems via retarded feedback," Journal of the Franklin Institute, 2103.

[22] H. Shen, S. Xu, J. Lu, and J. Zhou, "Passivity-based control for uncertain stochastic jumping systems with mode-dependent round-trip time delays," Journal of the Franklin Institute, vol. 349, no. 5, pp. 1665-1680, 2012.

[23] Z.-G. Wu, P. Shi, H. Su, and J. Chu, "Passivity analysis for discrete-time stochastic markovian jump neural networks with mixed time delays," IEEE Transactions on Neural Networks, vol. 22, no. 10, pp. 1566-1575, 2011.

[24] Z. Wu, P. Shi, H. Su, and J. Chu, "Asynchronous 12-1 filtering for discrete-time stochastic markov jump systems with randomly occurred sensor nonlinearities," Automatica. In press.

[25] H. Shen, X. Song, and Z. Wang, "Robust fault tolerant control of uncertain fractional-order systems against actuator faults," IET Control Theory \& Applications, vol. 7, no. 9, pp. 1233-1241, 2013.

[26] Z. Gait, M. S. de Queiroz, D. M. Dawson, and B. Xian, "Adaptive asymptotic tracking of parametric strict-feedback systems in the presence of additive disturbance," in Proceedings of the 43rd IEEE Conference on Decision and Control (CDC '04), pp. 11461151, December 2004.

[27] Z. Gai, M. S. de Queiroz, and D. M. Dawson, "Asymptotic adaptive regulation of parametric strict-feedback systems with additive disturbance," in Proceedings of the American Control Conference (ACC '05), pp. 3707-3712, June 2005.

[28] Z. Cai, M. S. de Queiroz, and D. M. Dawson, "Robust adaptive asymptotic tracking of nonlinear systems with additive disturbance," IEEE Transactions on Automatic Control, vol. 51, no. 3, pp. 524-529, 2006.

[29] B. Xian, D. M. Dawson, M. S. de Queiroz, and J. Chen, "A continuous asymptotic tracking control strategy for uncertain nonlinear systems," IEEE Transactions on Automatic Control, vol. 49, no. 7, pp. 1206-1211, 2004.

[30] Z. Cai, M. S. de Queiroz, and D. M. Dawson, "A sufficiently smooth projection operator," IEEE Transactions on Automatic Control, vol. 51, no. 1, pp. 135-139, 2006.

[31] M. Kristic, I. Kanellakopoulos, and P. V. Kokotovic, Nonlinear and Adaptive Control Design, Prentice-Hall, New York, NY, USA, 1995.

[32] P. A. Ioannou and J. Sun, Robust Adaptive Control, PrenticeHall, New Jersey, NJ, USA, 1996. 


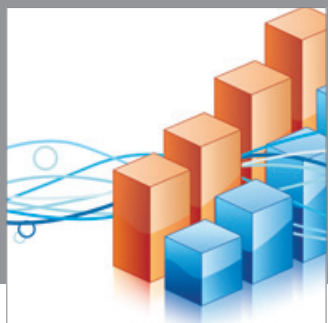

Advances in

Operations Research

mansans

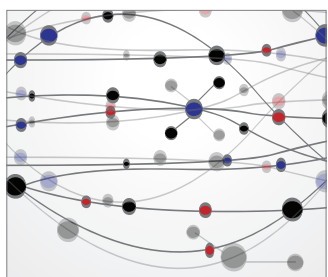

The Scientific World Journal
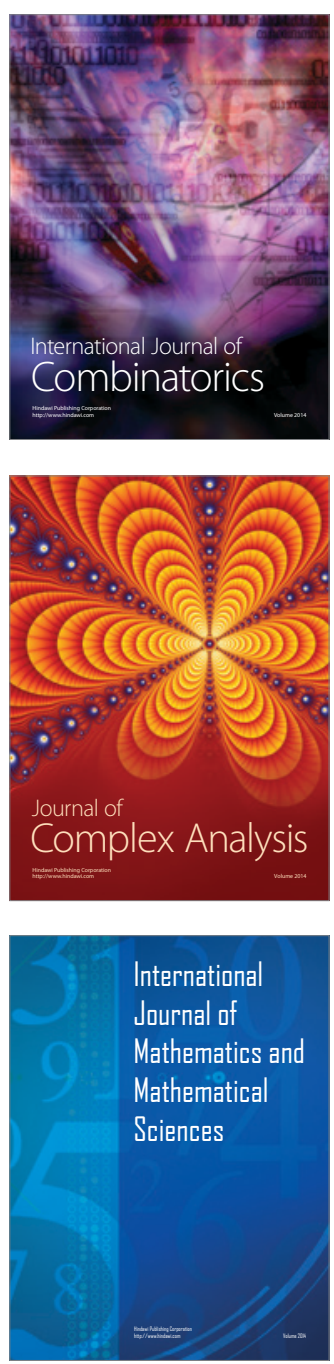


Submit your manuscripts at http://www.hindawi.com
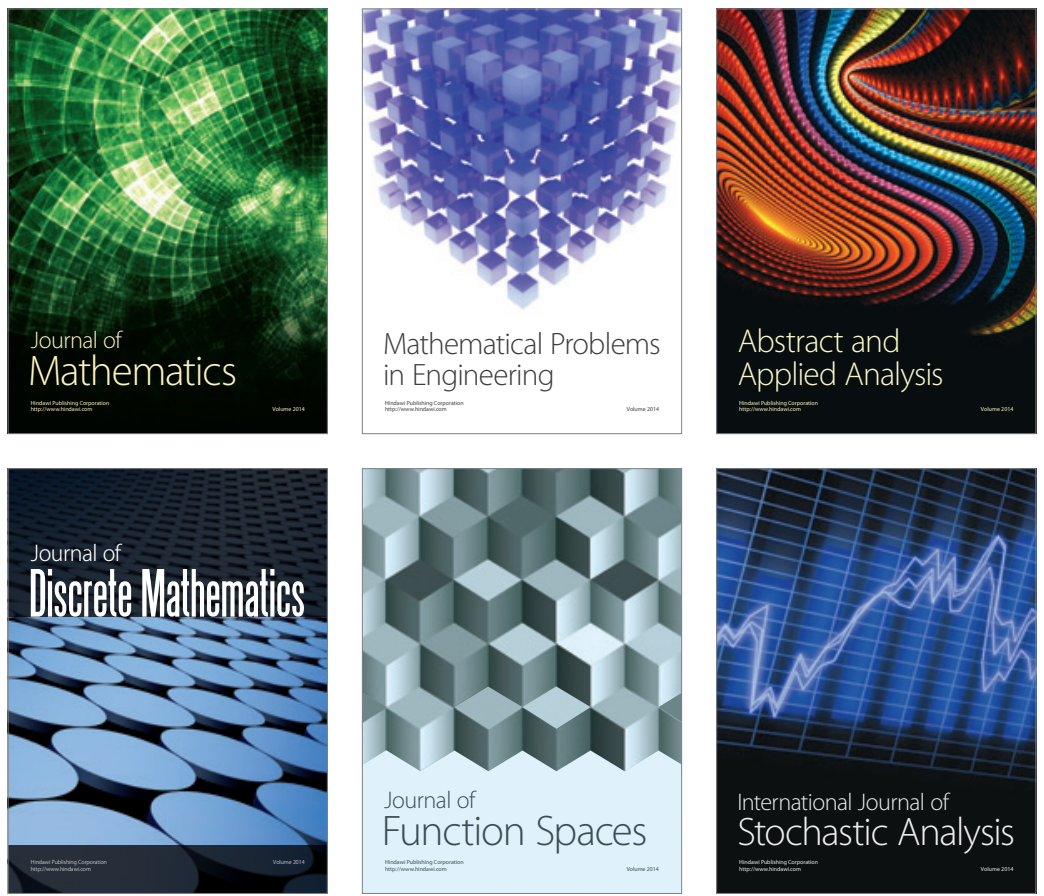

Journal of

Function Spaces

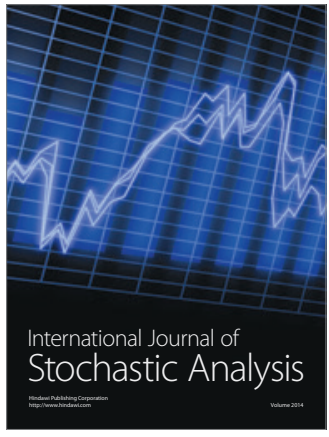

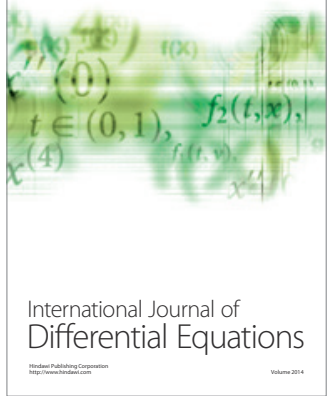
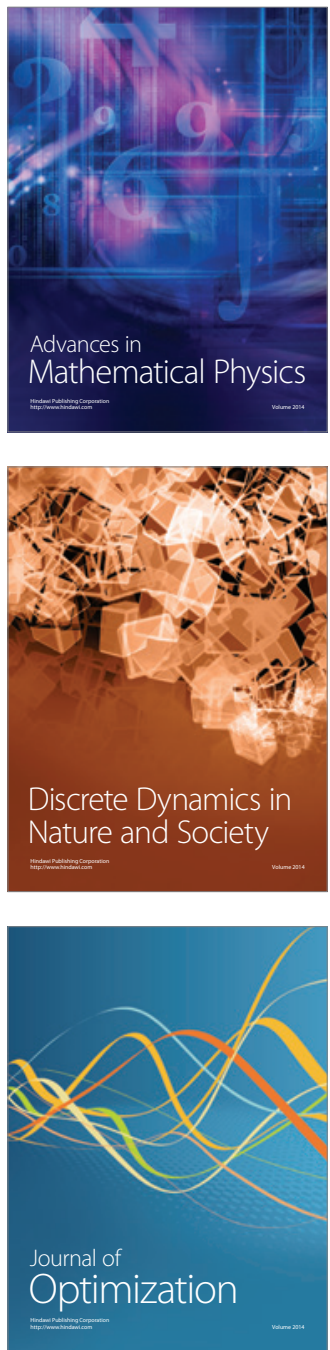